\title{
Are We There Yet? The Road to Gravitational Wave Detection
}

\author{
David E. McClelland ${ }^{\mathrm{A}}$ \\ A Centre for Gravitational Physics, Faculty of Science, Australian National University, \\ Canberra ACT 0200, Australia. Email: David.McClelland@anu.edu.au
}

Received 2004 August 20, accepted 2004 November 1

\begin{abstract}
Giant laser interferometers are currently 'listening' for gravitational waves but are they sensitive enough? I briefly review the status of the global effort to detect gravity waves and overview Australia's role.
\end{abstract}

Keywords: gravitational waves — instrumentation: interferometers

\section{Introduction}

The direct detection of gravitational waves (GWs) has been a major goal of fundamental physics since Weber's pioneering experiments (Weber 1969) with resonant bars in the 1960s. Giant laser interferometers are now setting upper limits on various astrophysical sources of gravitational waves. However, to guarantee detection and open the field of GW astronomy, even the most sensitive of the existing detectors will need to be improved by a factor of at least ten. In this overview, we will briefly review what has been achieved to date, the plans for improvement, and Australia's contribution to what is one of the most exciting scientific endeavors of this century.

\section{Laser Interferometry}

Whilst initial attempts to detect gravitational waves were based on sensing the ringing a passing wave would induce in a solid bar, the most promising technology is long baseline laser interferometry (Forward 1978; Saulson 1994). A passing gravitational wave will alternately stretch then contract one arm of a Michelson interferometer (Figure 1) whilst contracting then stretching the other arm. The problem is that the effect is extremely small: expressed as a relative length change, $\delta L / L$, it is less than $10^{-22}$.

As with the electromagnetic spectrum, the GW spectrum spans many orders of magnitude. Despite isolation systems which can virtually render the mirrors of the interferometer immune to seismic noise, Earth-based GW detectors cannot detect signals below about $10 \mathrm{~Hz}$ due to direct Newtonian coupling of the mirrors to moving matter. As GW arise from the bulk motion of massive objects, it is not expected that many sources will exist above about $10 \mathrm{kHz}$. Hence the goal with Earth-based detectors is to build an interferometer sensitive to GWs in the audio frequency band. The physical length of the interferometer is chosen as long as possible within the constraints of cost and Earth curvature; cavities are then used to store light in the arms to enhance the phase shift imposed on the light due to the optical path length change induced by a passing GW. For example, the Laser Interferometer

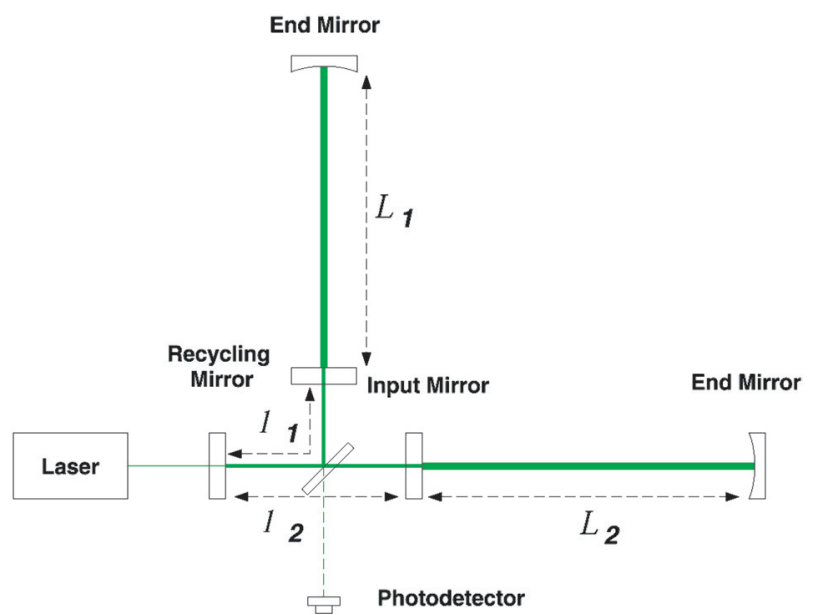

Figure 1 Optical layout of first-generation long baseline interferometers.

Gravitational-Wave Observatory (LIGO) interferometer (Abbott et al. 2003a), shown in Figure 2 (top), is $4 \mathrm{~km}$ long with the arm cavities having a finesse of the order of 200. This results in an instrument whose sensitivity, as shown in Figure 3 (noiseless line), rolls off above the corner frequency of the cavities, $100 \mathrm{~Hz}$. If the storage time of the optical cavity is fixed, the only way to improve the sensitivity at high frequencies is to increase the power on the beamsplitter, either by increasing the laser power or using power recycling (Drever 1983). The latter technique involves inserting a mirror (recycling mirror in Figure 1) before the beamsplitter to coherently reflect light exiting the interferometer toward the laser back into the interferometer - recycling the light.

At low frequencies (below about $40 \mathrm{~Hz}$ ), current instruments are limited by seismic noise transmitted through the imperfect isolation stacks. In the central region, the predicted limit is from thermal (Brownian motion) noise of the suspension system and test masses. The sensitivity of the $3 \mathrm{~km}$ Franco-Italian VIRGO interferometer (Acernese et al. 2004), shown in Figure 2 (bottom), and TAMA300 (Takahashi 2004) in Japan are subject to similar limitations 

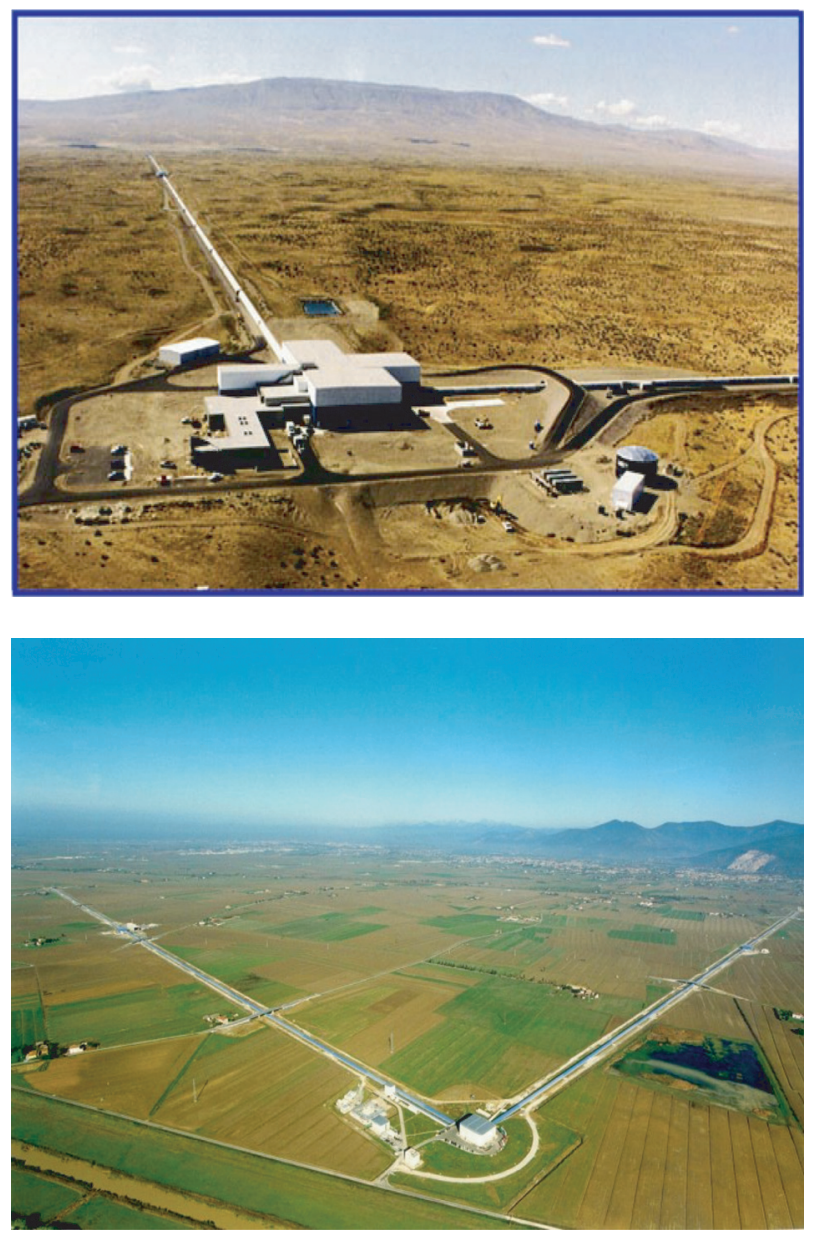

Figure 2 Top: LIGO Facility, Hanford, WA, USA. Bottom: VIRGO Observatory, Cascina, Italy.

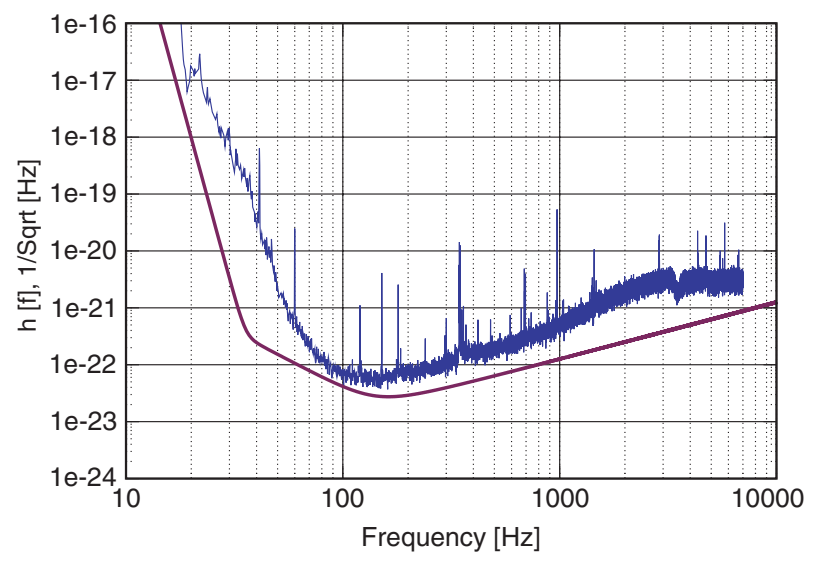

Figure 3 LIGO Hanford sensitivity curves. Line: design goal, noise curve: sensitivity, 2004 January 4.

although the frequency range over which different noise sources dominate may vary.

The other operating large-scale interferometer is GEO600 in Germany, a joint British-German project (Kotter et al. 2002). GEO600 employs a technique, called signal recycling (Meers 1988), which enables the shape of the sensitivity curve to be manipulated. Signal recycling allows bandwidth to be traded off against peak sensitivity and the frequency of maximum sensitivity to be adjustable. In this way, the shorter GEO instrument can reach similar sensitivity to the long baseline interferometers albeit over a narrow frequency range.

\section{Operating Detectors - Setting Upper Limits}

The design sensitivity for LIGO is shown in Figure 3, with VIRGO expected to perform at a similar level and GEO600 to be comparable in a narrow frequency range. By 2004 January, LIGO (see noise trace in Figure 3), GEO600 and TAMA300 had reached to within less than a factor of ten of their design goals. With such performance, whilst detection is not likely, it is possible to begin to improve on upper limits set by resonant bar detectors for GW emission from various astrophysical sources. Furthermore, such analyses enable shakedown of the process of combining data and candidate events from different interferometers network analysis. The most active in this endeavor is the LIGO Scientific Collaboration (LSC), which is analysing data from the three LIGO interferometers and GEO600. In addition, the LSC has embarked on joint analyses with TAMA300 and the ALLEGRO bar detector (Whelan et al. 2003) in Louisiana, USA.

Using early data from LIGO and GEO600 from the so called S1 data run, the LSC has published upper limits for GW emission from four source classes (Abbott et al. 2004b-2004e): burst sources such as supernovae (from $4 \mathrm{~ms}$ to $100 \mathrm{~ms}<1.6$ events per day at $90 \% \mathrm{CL}$ ), pulsars (for PSRJ1939, $h_{\max }<2 \times 10^{-22}$, ellipticity $<$ $\left.7 \times 10^{-5}\right)$, inspirals such as neutron stars $(<140$ events per year per MWEG), and stochastic background $\left(\Omega_{\mathrm{GW}}<50\right.$ between $40 \mathrm{~Hz}$ and $300 \mathrm{~Hz}$ ). Whilst these initial results are not of astrophysical interest there main importance is in the analysis techniques developed and the strategies used. As any signals present are buried in noise, data analysis methods such as matched filtering are essential to pushing the instrumental sensitivity.

Since $S 1$ there have been three further science runs (S2, S3, and S4). Data from these runs will set interesting limits and begin to constrain astrophysical models.

\section{Getting the Factor of Ten}

Once the LIGO VIRGO network is fully operational, the reach of the network (for neutron star-neutron star inspirals) will be out to the VIRGO cluster. It is possible that GW detection will be achieved; however, the event rate is likely to be less than one per year. Increasing the sensitivity of the long instruments by a factor of ten will increase the volume of the universe accessible by a factor of 1000 . Many GW events should then be recorded per day, finally igniting GW astronomy.

The factor of ten can be achieved by upgrading interferometer sub systems (see for example Gustafson et al. 1999). Active preisolation systems and new multiple pendulum isolation stacks will render seismic noise negligible (cut-off below direct Newtonian coupling). High quality fused silica suspension ribbons (already tested in GEO600), ultra high- $Q$ test mass substrates, probably 


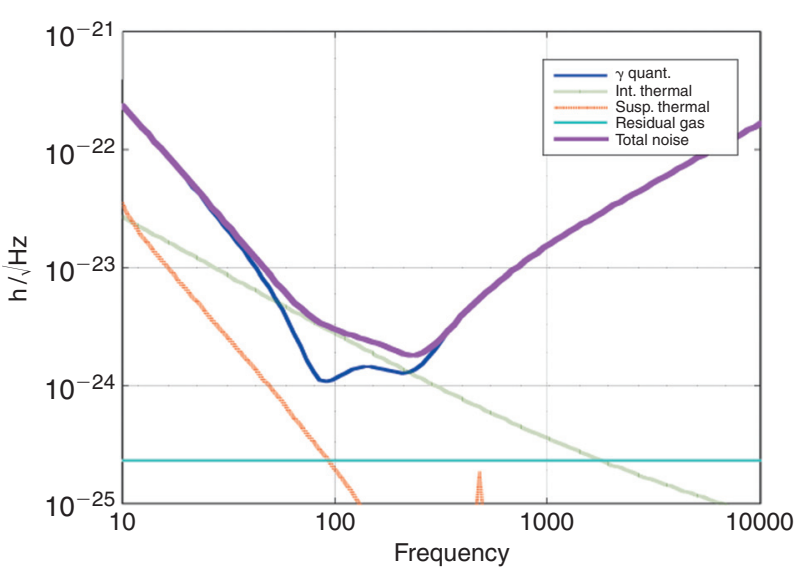

Figure 4 Predicted sensitivity spectrum for Advanced LIGO.

made from fused silica, and ultra low-loss-mirror coatings will reduce thermal noise by a factor of ten. Increasing the laser power to $150 \mathrm{~W}$ (from $10 \mathrm{~W}$ ) will reduce the photon shot noise, the dominant noise source at higher frequencies. Signal recycling will be employed to tailor the optical noise floor to maximize overlap with a specified GW waveform.

Figure 4 shows the design sensitivity for Advanced LIGO (Shoemaker 2003), the upgrade to LIGO planned to occur before the end of this decade. The Large-scale Cryogenic Gravitational Wave Telescope (Uchiyama et al. 2004) in Japan should reach a similar sensitivity using the conventional optical configuration, but cooling the suspension and mirrors to cryogenic temperatures to reduce thermal noise. The reach of a network of such instruments is predicted to be over $400 \mathrm{Mpc}$ for neutron star-neutron star inspirals.

\section{The Australian Consortium for Interferometric Gravitational Astronomy (ACIGA)}

The Australian Consortium for Interferometric Gravitational Astronomy consists of research groups from five universities: the Australian National University (ANU), the University of Western Australia (UWA), the University of Adelaide (UA), Monash University, and the University of Melbourne. The CSIRO's Space Optics Group is an affiliate member. ACIGA was established in 1995 with the near term goal to participate in GW detection and a long term goal to establish a full scale detector in Australia. ACIGA has research activities in the four major interferometer sub systems: data analysis; isolation, suspension, and thermal noise; lasers and optics; optical configurations.

The data analysis group at the ANU participated in setting up the LIGO Data Analysis System, and has ongoing research activities in characterizing the noise floor of LIGO, signal extraction, environmental noise correlations, global network analysis, and GW waveforms (Scott et al. 2004). The ACIGA Data Analysis Cluster is part of the LIGO Data GRID. Work on global networks will quantify the importance of a Southern Hemisphere site in a global
GW network in terms of sky coverage and source location (Searle, Scott, \& McClelland 2002).

The ACIGA group at UWA was one of the pioneers in the development of all metal vibration isolation systems. Their novel use of 'Euler springs' pushes modes of the isolation stack outside the frequencies of interest (Winterflood \& Blair 2000). They have developed a passive pre isolator (Garoi et al. 2003) which in conjunction with the isolator eliminates seismic noise in the signal band. A further innovation employs niobium flexure suspensions with sapphire test masses to significantly reduce suspension thermal noise. This technology is an alternative to the fused silica suspension under development by the LSC. They have conducted important research on sapphire to understand and reduce absorption and scattering (Yan et al. 2004).

ACIGA/UA is at the forefront of the development of high power, $100 \mathrm{~W}$ class, low noise, $\mathrm{CW}$ lasers (Mudge et al. 2002). Their stable/unstable resonator geometry and method of pumping the laser slab maximizes the conversion efficiency of diode pump light into the lasing mode. A $10 \mathrm{~W}$ laser developed at UA (Hosken et al. 2003) will soon be installed on the TAMA300 interferometer.

Advanced optical configurations and laser stabilization are the foci of the experimental research group at the ANU. Many elements of the control system for a signal recycled interferometer, developed and prototyped in the ANU laboratory (Shaddock et al. 2003), have been adopted for the Advanced LIGO baseline design. The optical system placed between the output of the interferometer and the photodetectors is referred to as the Output Optics system. ACIGA is poised to take the lead in developing and delivering this system to Advanced LIGO. Whilst signal recycling manipulates the interferometer response, quantum optics can be used to reduce the optical noise floor (McKenzie et al. 2002; de Vine, Gray, \& McClelland 2003). The development and use of 'squeezed light' for this purpose has been pioneered at ANU with the first production of squeezing in the audio frequency (GW) band recently reported (McKenzie et al. 2004).

In addition to the on campus research, ACIGA runs a research facility at a remote site in Gingin, Western Australia (see Figures 5 and 6). The current focus of the research at the Gingin facility is to diagnose the performance of a suspended optical system with of the order of $1 \mathrm{MW}$ of optical power circulating in it, power levels planned for Advanced LIGO. The first stage is nearing completion with the recent locking, in both length and alignment, of a low-power laser to an $80 \mathrm{~m}$ cavity (Slagmolen et al. 2004). This is a joint research project with LIGO and the LSC.

\section{Summary}

The performance of the current generation of large scale gravitational wave detectors demonstrates that interferometers can be built and operated with sensitivities and duty cycles approaching that needed to detect GWs. Ongoing research and development, both international, and within 


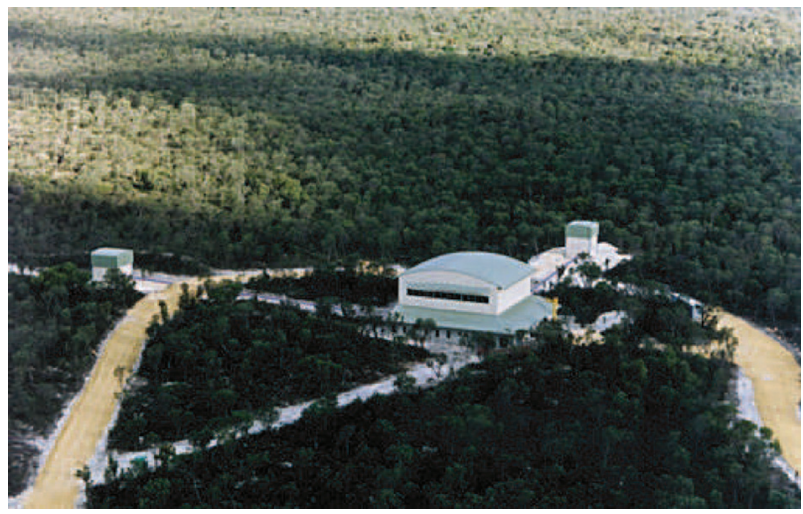

Figure 5 ACIGA's test facility at Gingin.

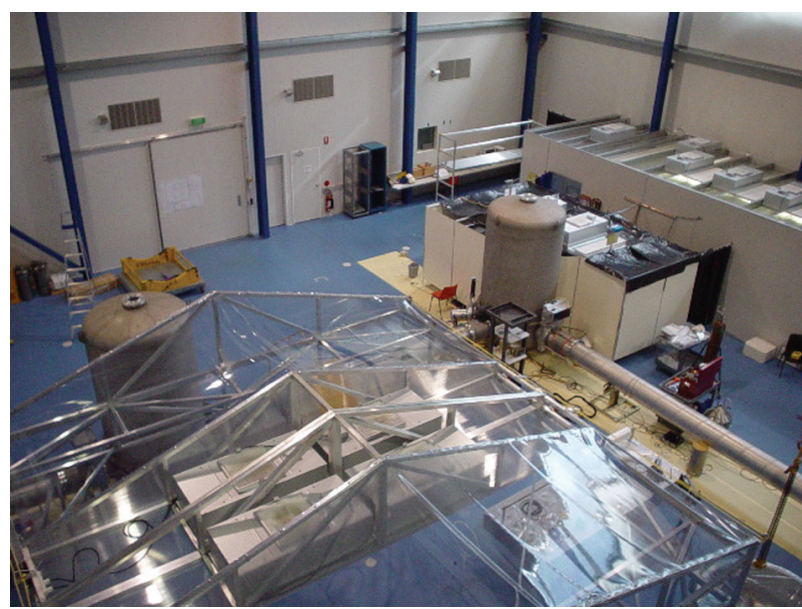

Figure 6 Inside the Gingin laboratory.

Australia is defining the technologies required to upgrade these detectors to ensure regular detection. It is anticipated that by the end of this decade these upgrades will be installed on full-scale interferometers. So are we there yet? No, but our destination is just over the next hill!

\section{Acknowledgments}

I thank my colleagues in GEO, LIGO, and the LSC, TAMA, and VIRGO for access to data and information.
I am indebted to my colleagues in ACIGA for their ongoing excellent research. I acknowledge the support of the Australian Research Council.

\section{References}

Abbott, B., et al. 2004a, NIMP, A517, 154

Abbott, B., et al. 2004b, PhRvD, 69, 082004

Abbott, B., et al. 2004c, PhRvD, 69, 102001

Abbott, B., et al. 2004d, PhRvD, 69, 122004

Abbott, B., et al. 2004e, PhRvD, 69, 122001

Acernese, F., et al. 2004, CQGra, 21, S385

de Vine, G., Gray, M. B., \& McClelland, D. E. 2003, PhLA, 316, 17 Drever, R. W. P. 1983, Gravitational Radiation (Amsterdam: North Holland), 321

Forward, R. L. 1978, PhRvD, 17, 379

Garoi, F., Winterflood, J., Ju, L., Jacob, J., \& Blair, D. G. 2003, RScI, 74,3487

Gustafson, E., et al. 1999, LSC White Paper on Detector Research and Development, www. Iigo.org/

Hosken, D. J., Mudge, D., Hollitt, C., Takeno, K., Veitch, P. J., Hamilton, M. W., \& Munch, J. 2003, PThPS, 151, 216

Kotter, K., et al. 2002, CQGra, 19, 1399

McKenzie, K., Shaddock, D. A., McClelland, D. E., Buchler, B. C., \& Lam, P. K. 2002, PhRvL, 88, 231102

McKenzie, K., Grosse, N., Bowen, W. P., Whitcomb, S. E., Gray, M. B., McClelland, D. E., \& Lam, P. K. 2004, PhRvL, 93, 161105

Meers, B. J. 1988, PhRvD, 38, 2317

Mudge, D., Ostermeyer, M., Ottaway, D. J., Veitch, P. J., Munch, J., \& Hamilton, M. W. 2002, CQGra, 19, 1783

Saulson, P. R. 1994, Fundamentals of Interferometric Gravitational Wave Detectors (Singapore: World Scientific)

Scott, S. M., Searle, A. C., Cusack, B. J., \& McClelland, D. E. 2004, CQGra, 21, S853

Searle, A. C., Scott, S. M., \& McClelland, D. E. 2002, CQGra, 7, 1465

Shaddock, D. A., Gray, M. B., Mow-Lowry, C., \& McClelland, D. E. 2003, ApOpt, 42, 1283

Shoemaker, D. 2003, CQGra, 20, S11

Slagmolen, B. J. J., et al. 2004, www . physics . uwa . edu . au / bram/gingin/aws.html

Takahashi, R. 2004, CQGra, 21, S403

Uchiyama, T., et al. 2004, CQGra, 21, S1161

Weber, J. 1969, PhRvL, 22, 1320

Whelan, J. T., Daw, E., Heng, I. S., McHugh, M. P., \& Lazzarini, A. 2003, CQGra 20, S689

Winterflood, J., \& Blair, D. G. 2000, PhL, 277, 143

Yan, Z., Ju, L., Eon, F., Gras, S., Zhao, C., Jacob, J., \& Blair, D. G. 2004, CQGra, 21, S1139 